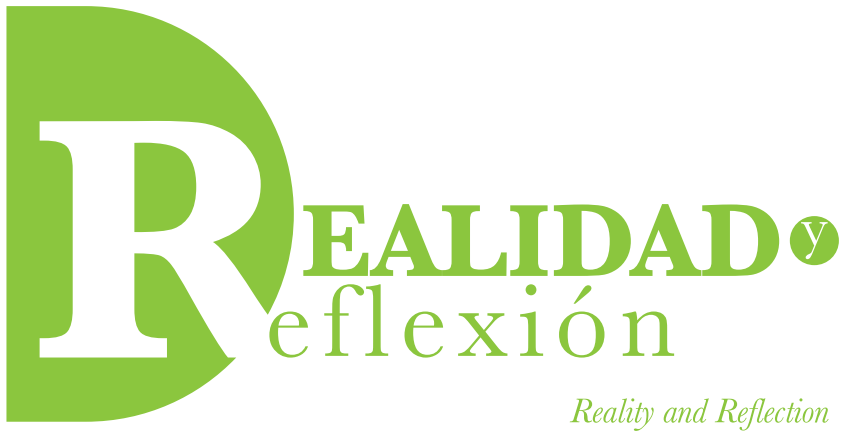

ISSN 1992-6510

Año 15, N 42, San Salvador, El Salvador, Centroamérica. Revista Semestral Julio-Diciembre 2015

Year 15, N 42, SAN Salvador, El SALVAdor, Central America. Semestral Journal July-December 2015

\title{
El mercado único digital será la clave del éxito para el comercio internacional en la Unión Europea
}

\author{
The digital single market is the key to success for \\ international trade in the European Union
}

\author{
Alicia Urquilla Castaneda \\ Máster en Creación y Dirección de Empresas de Nebrija Business School. \\ Licenciada en Administración de Empresas de la Universidad Dr. José Matías Delgado. \\ Investigadora Asociada, ICTI-UFG \\ aliciaurquicasta@gmail.com
}

\section{RESUMEN}

Europa está asumiendo la revolución digital y está tratando de ofrecer oportunidades digitales a través de la fuerza del mercado único de la Unión Europea. Este artículo abarca 16 iniciativas bajo tres pilares, proponiendo nuevas normas que faciliten el comercio electrónico. Además, se explica la visión que se tiene de esta iniciativa, los beneficios y los retos, la competencia con otros países, y las implicaciones en las PYMES. Al final se expondrán las conclusiones y las recomendaciones.

Palabras clave: Mercado único digital, Unión Europea, comercio digital, barreras digitales, comercio electrónico.

\section{ABSTRACT}

Europe is taking the digital revolution and is trying to offer digital opportunities through the power of the European Union single market. This article covers 16 initiatives under three pillars, proposing new rules to facilitate electronic commerce. In addition, the vision we have of this initiative, the benefits and challenges, competition with other countries and the implications for SMEs. Finally, conclusions and recommendations will be presented.

Keywords: Digital single market, European Union, digital commerce, digital barriers, e-commerce. 


\section{Siglas utilizadas en este artículo}

\begin{tabular}{ll}
\hline Siglas & Significado \\
\hline CDR & Comité de las Regiones Europeo \\
\hline CE & Comisión Europea \\
\hline CIP & Programa Marco de Innovación y Competitividad \\
\hline e-SENS & Servicios electrónicos europeos simples e interconectados \\
\hline EE.UU. & Estados Unidos \\
\hline EUR & Euros \\
\hline ICTI & Instituto de Ciencia, Tecnología e Innovación \\
\hline IVA & Impuesto del valor agregado \\
\hline MDU & Mercado Digital Único \\
\hline PSD & Servicios de pago en el mercado interior \\
\hline PYMEs & Pequeñas y medianas empresas \\
\hline TIC & Tecnologías de la información y la comunicación \\
\hline TTE & Transporte, telecomunicaciones y energía \\
\hline UE & Unión Europea \\
\hline UFG & Universidad Francisco Gavidia \\
\hline
\end{tabular}

\section{Introducción}

La clave para entender la Unión Europea siempre ha sido la política, pero desde su nacimiento, el principal factor determinante ha sido el económico. Es lo que propició que se generase el actual "mercado único", que permite un libre intercambio de bienes y servicios, así como la libre circulación de ciudadanos por todo el territorio de los estados miembros. Sin embargo, como la propia Unión reconoce, la legislación vigente hasta la fecha no reconoce de igual manera el creciente comercio electrónico, y se han propuesto no sólo cambiarlo, sino potenciarlo.

Los obstáculos que impiden un acceso igualitario a los servicios de internet podrían estar llegando a su fin en la Unión Europea. La nueva apuesta de la Comisión Europea es la creación de un mercado único digital que permita unas reglas comunes para unir el mosaico de mercados digitales que hay ahora. La iniciativa se encuentra dentro de la Agenda Digital, uno de los siete pilares de la Estrategia Europa 2020 (Ver Figura 1). ${ }^{1}$

La construcción de un mercado único digital es uno de los siete objetivos clave de la Agenda Digital Europea. $^{2}$

1 Europa 2020 es la estrategia de crecimiento de la UE para la próxima década.

2 Disponible en: http://ec.europa.eu/digital-agenda/ 


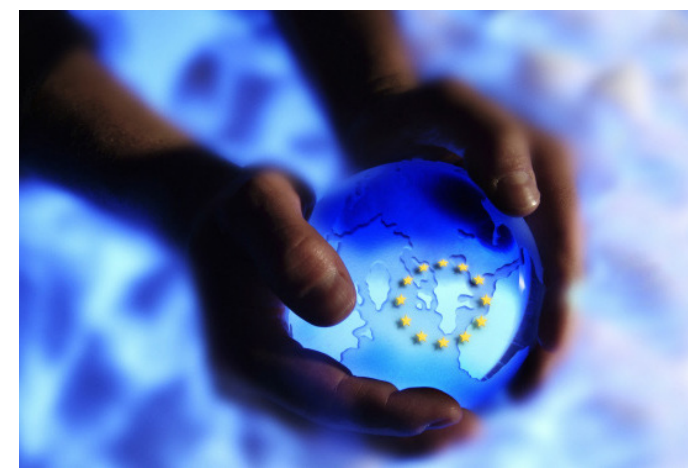

Figura 1.Mercado único digital dela Unión Europea

Fuente: cuadernosdeproduccion.blogspot.com.

Se basa en unificar la fragmentación existente entre los diferentes Estados miembros en lo relativo al pago por vía telemática a través de tarjeta de crédito, Internet o teléfono móvil, que son considerados los medios de pago con mayor potencial de mercado. La Comisión Europea elaboró el año pasado el Libro Verde "Hacia un mercado europeo integrado de pagos mediante tarjeta, pagos por Internet o pagos móviles", ${ }^{3}$ según el cual la integración de los mercados aportaría cuatro grandes ventajas: mayor competencia, mayor transparencia y posibilidad de elección para los consumidores, mayor innovación, y mayor seguridad de los pagos y confianza de los clientes. Durante el 2015 se trabajó en una serie de propuestas para alcanzar dicho objetivo que incluyen la revisión de la Directiva 2007/64/CE sobre servicios de pago en el mercado interior (PSD) y el Reglamento (CE) 924/2009 sobre los pagos transfronterizos en la Comunidad.

3 Disponible en: http://eurlex.europa.eu/LexUriServ/LexUriServ. do?uri=COM:2011:0941:FIN:ES:PDF
El comisario europeo de Mercado Interior y Servicios, Michel Barnier,señalaba recientemente que esta revisión de la normativa, realizada en el mes de julio, pretende promover el mercado único digital mediante el pago más barato y más seguro en Internet, tanto para los minoristas como para los consumidores. Los proveedores de servicios de pago electrónico estarán sujetos a los mismos estándares de regulación y supervisión que el resto de las entidades de pago, y tendrán la obligación de contar con un fuerte dispositivo de autenticación para todas las operaciones.

Así, los consumidores estarán también mejor protegidos contra el fraude, el abuso y los posibles incidentes de pago (el caso de operaciones ejecutadas deficientemente o impugnadas), y su responsabilidad si se detecta una operación con tarjeta de pago no autorizada se reduce a un máximo de 50 euros, frente a los 150 euros en que se situaba el límite antes de la revisión normativa.

Por otra parte, se está viendo la posibilidad de utilizar el teléfono móvil como dispositivo para facilitar las identificaciones online. Momentáneamente, Finlandia y Estonia son los únicos países europeos que ofrecen identificación en los pagos por móvil. Por consiguiente, se impulsará la aparición de nuevos actores y el desarrollo de métodos innovadores de pago a través de Internet y teléfonos móviles con objeto de impulsar la competitividad de la Unión Europea en el mundo. Según un estudio de la propia Comisión, el 36\% de los europeos accede ya a Internet a través de dispositivos móviles.

La Comisaria europea de Competencia, Margrethe Vestager, ha mencionado que no tiene 
sentido tener 28 regulaciones distintas junto a sus 28 organismos reguladores. Eso, obviamente, no ayuda a competir contra el resto como una unión, sino como un ente fragmentado. Un mercado digital bien regulado podría aportar 340,000 millones al producto interior de la Unión Europea, sin embargo existen barreras que impiden llegar a cifras así, tanto las impuestas de manera pública como las generadas por las propias empresas.

La Unión Europea demuestra que la competitividad está ligada más que nunca al comercio electrónico. Vestager ha propuesto abrir una investigación en el sector del comercio electrónico similar a las que se han hecho en el pasado en energía, servicios financieros o farmacéuticos, con el objetivo de encontrar las causas que actualmente frenan la competitividad y obstaculizan las ventas entre países miembros. Es un asunto que quiere tratar como máxima prioridad, poniendo el foco en los contratos de bienes y derechos sobre contenido digital, con gran importancia sobre los contratos de distribución. La investigación se llevará a cabo recogiendo información de muchas empresas de estados miembros, y a la larga les ayudará a ser más competitivos.

\section{Proyecto e-SENS}

España participa, junto con una veintena de países de la UE, en el proyecto e-SENS (Servicios electrónicos europeos simples e interconectados), impulsado por la Comisión Europea con el fin de desarrollar servicios públicos digitales transfronterizos, que facilitarán la creación de actividad, el cumplimiento de las obligaciones legales y la participación en las licitaciones.
La iniciativa pretende también contribuir al desarrollo del mercado único digital.

El proyecto e-SENS, vinculado al Programa Marco de Innovación y Competitividad (CIP) ${ }^{4}$, arrancó en abril de 2015 con un presupuesto de 27 millones de euros, la mitad de los cuales son aportados por la propia Comisión Europea, y en un periodo de ejecución de tres años. En su presentación, Neelie Kroes, vicepresidenta de la Comisión Europea, aseguró que, al permitir la interconexión de diferentes servicios públicos digitales existentes en Europa, los gobiernos podrán sacar el máximo provecho de sus estrategias digitales, las empresas podrán operar en el mercado único a un coste más bajo, se reducirán los trámites que los ciudadanos deberán realizar cuando se desplazan dentro de la UE.

\section{La visión de un mercado único digital europeo}

La Comisión Europea ha emprendido su plan de crear un "Mercado Único Digital" para América Latina. Las autoridades de la región han anunciado que durante 2015 y 2016 la Comisión Europea trabajará en 16 estrategias diversas con el objetivo de lograr un Mercado Único Digital para facilitar el e-commerce $e^{5}$ en el territorio.

El objetivo del mercado único digital radica en derribar los obstáculos reglamentarios, y pasar de 28 mercados nacionales a un mercado único. Se espera que una de las motivaciones principales de la comisaría sea facilitar el comercio online entre estados miembros de la unión, y ojalá fuera como muchos dicen también de que algo similar podría

\footnotetext{
4 Competitiveness and Innovation Framework Programme

5 Comercio electrónico.
} 
ocurrir con las llamadas, aunque de momento nada se sabe sobre qué pasará con el roaming ${ }^{6}$ de datos. Para facilitar las ventas online, ${ }^{7}$ el mercado digital único rebajaría los envíos entre países y agilizaría los trámites fiscales de estas compras.

De acuerdo con fuentes de la Comisión Europea, el objetivo es crear un Mercado Digital Único (MDU) que permita a las empresas y los consumidores aprovechar todo el potencial que ofrecería un espacio económico de 28 países y 500 millones de habitantes sin trabas físicas, tecnológicas, legales o fiscales. "La digitalización ha cambiado nuestro entorno pero el mercado europeo no funciona adecuadamente", dijo Andrus Ansip, vicepresidente de la Comisión Europea responsable de un proyecto que pretende modernizar y armonizar normas tan sensibles como las de propiedad intelectual, derechos contractuales de un contrato o procedimientos de adjudicación del espacio radioeléctrico.

El mercado único digital consiste fundamentalmente en eliminar las barreras nacionales a las transacciones efectuadas en línea. Se basa en el concepto de mercado común, concebido para eliminar las barreras comerciales entre Estados miembros de la Unión Europea.

Esta iniciativa es una continuación de la Estrategia de Lisboa, cuando la Estrategia Europa 2020 introdujo la Agenda Digital para Europa como una de las siete iniciativas emblemáticas, reconociendo el papel clave de capacitación que el uso de las tecnologías de la

6 Capacidad de un dispositivo para moverse de una zona de cobertura a otra.

7 En línea. información y la comunicación (TICS) tendrá que desempeñar para que la UE consiga sus ambiciones para el año 2020.

\section{Beneficios del mercado único digital en Europa}

En el marco de un encuentro hispano-sueco de telecomunicaciones organizado en Madrid, la empresa Ericsson afirmó que el desarrollo de un mercado digital único en la Unión Europea contribuiría con 415,000 millones de euros anuales a la economía europea y crearía centenares de miles de puestos de trabajo.

Según el presidente del CDR, Markku Markkula, la idea es "eliminar los obstáculos y mejorar el acceso de los consumidores a los bienes que se comercializan en línea". Dicen las fuentes de la Comisión Europea que "el objetivo es aumentar la prosperidad económica y contribuir a una unión cada vez más estrecha entre los pueblos europeos" y desarrollado posteriormente en el concepto de mercado interior, definido como "un espacio sin fronteras interiores, en el que la libre circulación de mercancías, personas, servicios y capitales estará garantizada”.

Otros beneficios, de acuerdo con las fuentes oficiales de la $\mathrm{CE}$, es que en cuanto a medios políticos específicos, la computación en nube, por ejemplo, permitiría que un $80 \%$ de organizaciones redujeran sus costes entre el $10 \%$ y el $20 \%$. Pueden citarse otros beneficios, como una mejora del teletrabajo (46\%), de la productividad (41\%) y de la normalización (35\%), así como nuevas posibilidades de negocio (33\%) y de mercados (32\%). Las personas vulnerables (personas de más edad, con movilidad reducida, aisladas en zonas rurales, 
con bajo poder adquisitivo) pueden obtener beneficios especiales del mercado único digital; la UE estará así mejor situada para abordar los retos demográficos actuales.

\section{Retos para conseguir un mercado digital único}

Algunas personas objetan que lo más complejo será armonizar 28 mercados, el pago de impuestos y leyes contractuales, que existe una alta probabilidad de que varios Estados miembros sean evasivos a pasar a un mercado único digital y que podría tratarse de medidas proteccionistas.

Por otro lado, el Comité de las Regiones Europeo (CDR) sostiene que aunque los planes para crear un mercado único digital son un paso en la buena dirección, resultan insuficientes para afrontar el problema de la brecha digital en Europa. De acuerdo con los representantes del mencionado organismo, las propuestas pasan por alto "la importancia que tienen los entes locales y regionales en el desarrollo de las infraestructuras de banda ancha, así como el papel que desempeñan en la administración electrónica y a la hora de fomentar la utilización de datos masivos para mejorar los servicios públicos y privados. $\mathrm{El} \mathrm{CDR}$ pide que se presenten ideas e iniciativas más concretas para nuevas inversiones y advierte de que las propuestas no son suficientemente ambiciosas para superar las divergencias existentes entre las normativas de los Estados miembros".

El líder de la institución cree que parece estar ignorándose que "la dimensión territorial podría exacerbar las divisiones existentes en el acceso a Internet". Es decir que uno de los principales retos de Europa es conseguir que todos los ciudadanos tengan un acceso a Internet, por lo que será necesario "luchar contra las disparidades".

Bruselas considera necesario que los Estados miembros y sus instituciones compartan la responsabilidad de actualizar y aplicar los cambios de las directivas, y señalan como primordial permitir que los pagos online sean posibles tanto para pequeñas como medianas empresas, incidiendo en la importancia de la credibilidad y la confianza en los consumidores.

\section{Competir con las grandes firmas de Estados Unidos}

Según fuentes de la Comisión Europea, también se plantea revisar toda la legislación sobre telecomunicaciones, con la intención de algunos comisarios de aplicar esas normas a las llamadas plataformas digitales (como Facebook, Skype, Twitter), que están operando con obligaciones mucho menos estrictas que las operadoras telefónicas tradicionales.

Adicionalmente, el comisario europeo de Economía Digital, Günther Oettinger, afirmó en Cannes que "Europa necesita un mercado digital único que la proteja de los gigantes mundiales de la red como Amazon, Google o Microsoft". De acuerdo con el comisario, este mercado único digital aportaría una hegemonía a la región frente a los gigantes del mercado ya mencionados y llegados desde los Estados Unidos.

Se ha identificado que en los últimos años estas grandes empresas llegadas de América pagan impuestos en los mercados que les ofrecen más ventajas. Por ejemplo, firmas como Apple o Amazon tributan en Irlanda o Luxemburgo lo 
que venden en España, lo que les supone un gran ahorro en pagos de impuestos. El mercado único digital, haría frente a esto.

\section{La estrategia sobre el mercado único digital de la UE}

La estrategia consiste en el plan de la UE de crear un mercado único digital libre y seguro en el que los ciudadanos puedan comprar en línea a través de las fronteras, y las empresas puedan vender en todo el territorio de la UE con independencia del lugar de la UE en que residan. Su objetivo es ampliar la economía digital de la UE para ofrecer a los consumidores mejores servicios a mejores precios y ayudar a las empresas a expandirse.

\section{En la Comisión}

La estrategia, publicada por la Comisión el 6 de mayo de 2015, tiene tres objetivos:

- Facilitar a consumidores y empresas el acceso en línea a productos y servicios de toda Europa.

- Mejorar las condiciones para que las redes y servicios digitales crezcan y prosperen.

- Impulsar el crecimiento de la economía digital europea.

La necesidad de esta estrategia es que los ciudadanos europeos se enfrentan a obstáculos cuando utilizan servicios y herramientas en línea. Los mercados en línea siguen siendo nacionales en buena medida: solo un $15 \%$ de los ciudadanos compran en línea de otro país de la UE y únicamente un $7 \%$ de las pequeñas y medianas empresas venden en otro país de la UE. Las barreras reglamentarias persistentes y la fragmentación del mercado dan pie a que los ciudadanos no puedan aprovecharse de bienes y servicios y a una reducción en el crecimiento de las empresas.

Para desbloquear este potencial la UE planea realizar reformas profundas que van desde un nuevo marco de los derechos de autor a la entrega de paquetes y las telecomunicaciones.

Para ver cómo está diseñada la estrategia del mercado único digital de la UE, se explica en detalle a continuación.

\subsection{Un mercado único digital de Europa-COM (2015) 192}

La estrategia de la Comisión para el mercado único digital incluye varias medidas, tanto legislativas como no legislativas que se irán haciendo públicas en 2015 y 2016. Se dividen en tres pilares:

- Mejorar el acceso a los productos y servicios en línea.

- Mejorar las condiciones para que las redes y servicios digitales crezcan y prosperen.

- Impulsar el crecimiento de la economía digital europea.

\subsubsection{Mejorar el acceso a los productos y servicios en línea}

Reforma de las normas para las ventas transfronterizas en línea

Esto incluye una propuesta de la Comisión para armonizar las normas de la UE en materia de adquisición de contenidos digitales, como 
aplicaciones o libros electrónicos, así como los derechos a nivel de la UE para las ventas transfronterizas en línea de bienes físicos y la mejora de los derechos de los consumidores.

\section{Mejora de la paquetería transfronteriza}

Para ello se introducirán medidas destinadas a mejorar la transparencia en materia de precios y la vigilancia regulatoria del mercado transfronterizo de paquetería.

\section{Fin del geobloqueo}

La Comisión propondrá un proyecto de ley para poner fin al llamado "geobloqueo", esto es, la denegación de acceso a sitios de internet basados en otro país de la UE o que se cobren precios distintos en función de la localización de un cliente.

Reforma de la legislación europea sobre derechos de autor

La Comisión propondrá medidas que garanticen que los servicios de contenido, como los servicios de vídeo, puedan utilizarse en otros países. Gracias a ello, las personas que hayan comprado una película o música en su país podrán seguir disfrutando de ella cuando viajen por Europa. La Comisión también intensificará los esfuerzos en contra de las infracciones en materia de derechos de propiedad intelectual.

Reducción de la burocracia relacionada con el IVA Esto incluye medidas que permitan a los vendedores de bienes físicos beneficiarse del registro y pago electrónicos únicos y de la introducción de un umbral común del IVA que ayude a las nuevas empresas innovadoras a trabajar en línea.

\subsubsection{Mejorar las condiciones para que las redes y servicios digitales crezcan y prosperen}

Reforma de las normas de telecomunicaciones de la UE

Esto incluye medidas que propondrá la Comisión:

- Facilitar la coordinación de la gestión del espectro.

- Fomentar las inversiones en banda ancha de alta velocidad.

- Mejorar el marco reglamentario institucional.

Revisar las normas en materia de medios audiovisuales

Para ello se revisará la Directiva de servicios de comunicación audiovisual, y se introducirán medidas en materia de promoción de las obras europeas y normas para la protección de los menores y publicidad.

Evaluación de la función de las plataformas en línea Ello implicará una evaluación por parte de la Comisión en relación con la falta de transparencia de los resultados de las búsquedas, el uso que hacen las plataformas en línea de la información que recogen, las relaciones entre plataformas y proveedores y las restricciones de la posibilidad que tienen las personas de pasar de una plataforma a otra. También se analizarán las formas de abordar los contenidos ilegales en Internet.

Impulso de la confianza en el tratamiento de los datos personales en el contexto de los servicios digitales Esto incluye una posible revisión de la Directiva sobre el derecho a la intimidad y 
las comunicaciones electrónicas que se aplica en la actualidad únicamente a las compañías tradicionales de telecomunicación y no afecta a otros proveedores de servicios digitales. La revisión de la Comisión se llevará a cabo tras la entrada en vigor de las nuevas normas de la UE sobre protección de datos.

Esto incluirá también el establecimiento de una colaboración público-privada en materia de ciberseguridad.

\subsubsection{Impulsar el crecimiento de la economía digital europea}

\section{Construir una economía de los datos}

\section{La Comisión presentará:}

- Una iniciativa europea sobre libre circulación de datos para eliminar los obstáculos no relacionados con la protección de los datos personales que obstaculizan la libre circulación de datos en toda la UE.

- Una iniciativa europea sobre computación en nube, incluidos los servicios de certificación de la nube.

\section{Definición de las prioridades para normas e interoperabilidad}

Esto se centrará en ámbitos considerados fundamentales para el mercado único digital, como la sanidad electrónica, la planificación de desplazamientos, electrónico o la medición inteligente de la energía.

Creación de un mercado único digital inclusivo Se introducirán medidas para impulsar las destrezas digitales de la población, que la Comisión incorporará en futuras iniciativas sobre destrezas y formación.

También un nuevo plan de acción sobre administración electrónica para 2010-2020. Como parte de dicho plan, la Comisión:

- Pondrá en marcha un proyecto piloto para probar el principio de "presentación única" que garantiza que las administraciones públicas ponen en común determinados datos a nivel interno, de tal manera que no tengan que volver a pedirlos cuando tienen que volverlos a utilizar.

- Tratarán de interconectar los registros mercantiles en toda la UE.

- Se propondrán la creación de un "portal digital único", integrando varios portales y servicios europeos existentes.

\section{En el Consejo Europeo}

Por parte del Consejo Europeo, en su reunión del 25 y 26 de junio de 2015, los dirigentes de la UE respaldaron la estrategia y declararon que debería utilizarse para promover un crecimiento inclusivo en todas las regiones de la UE. Instaron a que se actuara para hacer frente a la fragmentación del mercado, construir la infraestructura digital necesaria y fomentar la digitalización de la industria europea. También solicitaron una rápida adopción de nuevas normas en materia de telecomunicaciones, ciberseguridad y protección de datos.

\section{En el Consejo}

Los ministros de Cultura respaldaron la portabilidad transfronteriza de los contenidos, la 
lucha contra los contenidos ilegales y la necesidad de encontrar un equilibrio adecuado en materia de derechos de autor. También declararon que el principio de territorialidad es importante para la creación de contenidos, lo que significa que el geobloqueo debe reducirse, pero que puede justificarse en algunos casos.

Estos ministros de Cultura acordaron que la Directiva de servicios de comunicación audiovisual existente debía adaptarse a la evolución tecnológica. En esta revisión era preciso tomar en cuenta lo siguiente:

- Garantizar la libertad de los medios de comunicación y la promoción de la diversidad cultural.

- Mantener el principio del país de origen (cada proveedor de servicios audiovisuales queda bajo la jurisdicción de un único Estado miembro).

- Racionalizar el procedimiento que siguen los Estados miembros cuando se convierten en objetivo de contenidos inaceptables procedentes de otro Estado miembro.

Los ministros de Competitividad estuvieron de acuerdo en que la estrategia abarque todas las cuestiones más importantes para crear el mercado único digital. Destacaron la importancia de crear normas en materia de derechos de autor adecuadas para sus fines, actualizando las normas de comercio electrónico, impulsando la confianza de los consumidores, la sensibilización y la protección, e insistieron en la necesidad de actuar para reforzar las destrezas digitales y las normas sobre protección de datos. También definieron varias acciones prioritarias:

- Crear las condiciones adecuadas para las pequeñas y medianas empresas, en especial las de reciente creación.

- Promover la digitalización de la industria europea.

- Aplicar y ampliar la administración electrónica en las administraciones públicas.

- Impulsar las inversiones en las infraestructuras $\mathrm{y}$ redes digitales.

- Evaluar las repercusiones de las normas fiscales en los instrumentos digitales.

- Considerar el principio de "digital por defecto" para toda la nueva legislación de la UE.

Los ministros también estuvieron a favor de la creación de un programa europeo de ciencia abierta, que facilitaría el acceso a las publicaciones científicas financiadas con fondos públicos y a otros datos afines. Declararon que la investigación debería desempeñar un papel más importante en la estrategia del mercado digital y contribuir a la innovación digital.

Los ministros de Telecomunicaciones debatieron también la estrategia del mercado único digital en su reunión de Consejo TTE de los días 11 y 12 de junio de 2015. Acogieron con satisfacción sus objetivos y reiteraron la importancia de una economía digitalizada para impulsar el empleo y el crecimiento y fomentar la competitividad de la UE. 
Entre los aspectos que destacaron los ministros se incluían:

- El papel de la digitalización en la estimulación del emprendimiento y el crecimiento de las PYMES.

- La necesidad de reforzar la ciberseguridad e impulsar la confianza en los servicios electrónicos.

- La necesidad de mejorar la infraestructura y el acceso a la banda ancha para todos los ciudadanos.

- La necesidad de que la tarificación de los servicios de paquetería sea más transparente en toda la UE.

Los ministros destacaron también que era conveniente una buena coordinación a nivel nacional para garantizar que la estrategia se aplica efectivamente.

Los ministros de Justicia, el 15 de junio alcanzaron una orientación general sobre el Reglamento de protección de datos. Esto ha permitido al Consejo y al Parlamento Europeo dar comienzo a las negociaciones. El Reglamento mejorará la protección de los datos personales a la vez que mejora las oportunidades para las empresas en el mercado único digital.

\section{Las PYMES online impulsan el crecimiento de las exportaciones en Europa}

La influencia por el comercio online ha permitido impulsar el crecimiento de numerosas PYMES europeas y obtener unas ventas por valor de cerca de 18,500 millones de euros en los últimos cuatro años. Así lo revela un estudio realizado por eBay Public Policy Lab y el equipo de economistas de Sidley Austin LLP, que analiza los datos de ventas de eBay durante el periodo 2010-2014 y el número de PYMES que están presentes en la plataforma de e-commerce.

El estudio indica que las exportaciones intraeuropeas de las PYMES europeas presentes en eBay se incrementaron un 61\% entre 2010 y 2014, porcentaje que cuadruplica la tasa de crecimiento del comercio tradicional, y el número de PYMES europeas que exportaron a través de eBay a 15 o más países creció un 48\%. En 2014, alrededor del 93\% de las PYMES online que vendieron a través de eBay exportaron, frente al $26 \%$ de los comercios tradicionales.

Los cinco países europeos más avanzados a la hora de exportar, puesto que cuentan con más PYMES online globales, son Reino Unido (un 51,8\% del total), Francia (un 50,6\%), España (41,9\%), Italia (34,6\%) y Alemania (19,6\%). No obstante, las PYMES online alemanas son las que lideran el comercio intraeuropeo, con 15 puntos porcentuales por encima de la media europea, situada en el 39,1\%. Estas cifras reflejan que el e-commerce está ayudando a crear un modelo económico más inclusivo, que facilita un alcance global a pequeñas y medianas empresas en toda Europa.

En este sentido, la Comisión Europea, en su documento sobre estrategia del mercado único digital de mayo de 2015, reveló que eliminar las barreras al comercio online dentro de Europa podría suponer una contribución adicional al PIB europeo de 415 billones de euros. 
Paul Todd, vicepresidente de eBay en Europa, asegura que "muchas PYMES han salido reforzadas de la recesión y alcanzado públicos más globales. Con el crecimiento del comercio europeo, vemos el potencial que tiene para los negocios online la apertura completa del mercado único, que contemple medidas como la eliminación de las barreras administrativas y una mejora de los servicios de entrega. En este sentido, las nuevas tecnologías y las compañías como eBay están poniendo a disposición de los pequeños empresarios herramientas, antes reservadas para los grandes negocios, de las que pueden beneficiarse desde sus inicios como empresa”.

El ranking ${ }^{8}$ de eBay de países con más PYMES online que exportan a cuatro continentes lo lidera Reino Unido, seguido de Francia, España, Italia y Alemania.

\section{El comercio digital y sus implicaciones}

Para Bruselas el objetivo básico es lograr un mercado digital con menos fronteras y los mismos derechos y deberes en todo el territorio de la UE. Pero que a cada uno de los implicados en el comercio digital, espera Bruselas le serán aportados beneficios distintos.

Consumidores. La comisión cree que, tras la aplicación de las medidas anteriores, los consumidores estarán más protegidos a la hora de comprar por Internet y tendrán un abanico más amplio en las ofertas al haber entrado al mercado más empresas. Se bajarán los precios de distribución y entrega para las empresas lo que repercutirá en el bolsillo del destinatario final.

8 Clasificación
PYMES y start-ups.9 Con medidas como la eliminación del bloqueo geográfico, la Comisión pretender facilitar la entrada en el sector a pequeñas empresas. La propuesta de rebajar el IVA beneficiará a los pequeños negocios, pues el precio final del producto será más atractivo para el comprador.

El sector creativo. Con la unión digital, el mercado no solo se amplía y diversifica sino que se facilita la compraventa entre los 28 países de la UE. Habrá, según Bruselas, unas reglas más justas para todas las partes, incluyendo las intermediarias, mediante el control de la protección del copyright ${ }^{10}$ en todo el territorio comunitario. De esta forma también se combatirá la piratería.

Industria. Tendrán ahora un camino más regulado a la hora de operar sin incurrir en ilegalidades y existirá más competencia. Habrá inversión pública para desarrollar un programa de ciberseguridad del que se beneficiaría la industria digital. Y, por último, la Comisión opina que se elevarán los estándares en cuanto a la interoperabilidad, la velocidad y la innovación digital.

\section{Conclusiones}

Según la UE, la implantación de un marco único regulatorio para el comercio online podría contribuir en 415 mil millones de euros al año para la economía y crear 3,8 millones de puestos de trabajo. Y lo cierto es que los primeros movimientos de este nuevo mercado, sobre todo en lo que se refiere a ser equiparado al mercado

9 Empresas emprendedoras

10 Derechos de autor 
físico en muchos aspectos, han encajado muy bien en el ideario de los consumidores europeos.

Actualmente, y según los datos que maneja la Comisión, las barreras del mercado digital suponen una pérdida de oportunidades para los actores económicos europeos, consumidores y empresas. Ahora mismo, solo el 7\% del total de las PYMES del mercado único que opera en la red son transfronterizas, es decir, venden sin distinción en el total de la UE, lo que supone que las empresas de Internet asentadas y las emergentes no pueden aprovechar al máximo las oportunidades que ofrece operar en Internet en pleno 2015. El objetivo del mercado único digital es derribar estos muros reguladores que, a pesar de estar en el mismo espacio comunitario, cierran sus fronteras en materia de comercio en línea, de forma que la UE quiere pasar de 28 mercados nacionales a uno solo: un mercado único digital completamente funcional.

Dar el paso de 28 regulaciones comerciales a una única comunitaria. Por ello, la estrategia para el mercado único digital incluye un conjunto de acciones dirigidas a terminar con esta situación a través de tres pilares para unificar el mercado: un mejor acceso para los consumidores y las empresas a los bienes y servicios digitales en toda Europa, la creación de las condiciones adecuadas para que se dé una igualdad de condiciones en las redes digitales y servicios, y maximizar el potencial de crecimiento de la economía digital. Esto encierra, un conjunto de normas armonizadas sobre los contratos y la protección del consumidor para las compras en línea, tanto si se trata de bienes físicos como digitales, por ejemplo libros electrónicos o aplicaciones. Además, una de las pretensiones de la comisión para el conjunto del mercado es la de hacer asequible la entrega de paquetes y los servicios de mensajería, pues actualmente el $62 \%$ de las empresas que venden online creen que los costes de entrega son tan altos que en la mayoría de ocasiones suponen una barrera comercial.

Pero sin duda, una de las razones fundamentales que justifican la implantación del mercado único es terminar con los bloqueos regionales (Hola de nuevo, año 2015). El bloqueo regional, impuesto sobre por los market places ${ }^{11}$ de servicios y contenidos digitales (tiendas de $a p p s^{12}$, tiendas de consolas, servicios de contenidos en streaming... ${ }^{13}$ ), es una práctica discriminatoria utilizada por razones comerciales que se da, principalmente, cuando los vendedores de contenidos online impiden a los consumidores acceso a un sitio web o servicio en función de su ubicación (siempre dentro de la UE) o, y aquí viene lo importante, ajustan los precios en función del mercado local en el que se van a distribuir los bienes, teniendo en cuenta que se trata, generalmente, del mismo bien digital en funciones y contenidos, algo que también es extensible a bienes y servicios físicos.

Evidentemente, las barreras comerciales online en la UE son una de las lacras que están frenando el comercio en línea y que generan desigualdades y sesgos en función del país de origen y destino, algo que, en pleno 2015 -sobre todo para bienes y servicios online-, no puede tener cabida.

\footnotetext{
11 Lugares de mercado.

12 Aplicaciones.

13 Utilizado principalmente para proporcionar almacenamiento de copia de seguridad.
} 
Para evitar que todo el entorno comercial y cultural de los europeos esté en manos de empresas estadounidenses, la Comisión anuncia 16 medidas que propondrá durante los próximos dos años. En primer lugar, las iniciativas legislativas necesarias para armonizar las normas sobre protección del consumidor, para facilitar el comercio electrónico transfronterizo, una posibilidad que ahora prácticamente no se utiliza en Europa. Bruselas también intentará limar las diferencias entre las normas de copyright o propiedad intelectual, que a menudo impiden la descarga de bienes digitales (desde películas a música o aplicaciones) de un país a otro.

En ese mismo terreno, la $\mathrm{CE}$ se propone simplificar las normas sobre aplicación del IVA en operaciones transfronterizas, en las que se aplica el tipo del país del consumidor. Bruselas quiere permitir que las pequeñas y medianas empresas apliquen el tipo de imposición de su país para evitarles el papeleo de operar con tipos diferentes en cada mercado nacional.

La Comisión también plantea revisar toda la legislación sobre telecomunicaciones, con la intención de algunos comisarios de aplicar esas normas a las llamadas plataformas digitales (como Facebook, Skype, Twitter), que ahora operan con obligaciones mucho menos estrictas que las operadoras telefónicas tradicionales.

La Comisión Europea también estima que la mano de obra en el marco del mercado digital se incrementaría de 1.6 millones de empleados en 2013 hasta 4.8 millones en 2018, y triplicándose los ingresos, de 17,500 a 63,000 millones de euros.
Si Internet nos ofrece grandes oportunidades tanto a los ciudadanos como a las empresas, y si las nuevas tecnologías llevan a la innovación, es importante eliminar los obstáculos existentes en el ámbito digital entre los países europeos creando un mercado único digital conectado $y$, por tanto, una Europa digital, más dinámica, más abierta y más competitiva para conseguir más crecimiento y más empleo. Europa necesita crear las condiciones adecuadas para que las redes digitales y las empresas innovadoras puedan crecer a través del mercado único digital.

La Agenda Digital aborda aspectos esenciales de la economía moderna como el acceso al internet de alta velocidad y a los contenidos digitales, la seguridad informática, la eficiencia de los servicios públicos electrónicos y los nuevos servicios de salud que facilitan la vida a los ciudadanos. Además, trata de garantizar que todos los ciudadanos estén capacitados para sacar provecho de la revolución tecnológica. La "Asociación público-privada para la internet del futuro" es un programa europeo de investigación e innovación cuyo objetivo es impulsar la competitividad de la UE en relación con las tecnologías del internet del futuro y los sistemas para aplicaciones y servicios inteligentes. Asimismo, ayudará a las empresas y las administraciones públicas en el desarrollo de nuevas soluciones del internet basadas en datos complejos en línea para dotar de inteligencia a las infraestructuras y los procesos empresariales.

Otro objetivo de la Agenda Digital para Europa es lograr que el Internet sea más seguro para los menores. Tras hacer suyo ese objetivo, las principales empresas tecnológicas y de medios 
de comunicación establecieron una coalición para proteger a los usuarios infantiles de la red.

A la hora de lanzar la propuesta, la Comisión asegura tener en mente al consumidor, el gran beneficiado junto con las PYMES y la industria. La mayoría de las iniciativas están enfocadas a ampliar la oferta y sus posibilidades y aumentar su protección a la hora de comprar por Internet. Una bajada de los precios de distribución también supondría una bajada en el precio del producto final.

Las pequeñas y medianas empresas podrían tener un camino más fácil para acceder al universo digital. Solo un 7\% realizan ventas transfronterizas en el territorio europeo, lo que actualmente conlleva una media de 9,000 euros de costo adicional para adaptarse a las leyes de otros países, según advierte la Comisión. La propuesta de armonizar el IVA (y bajarlo) también beneficiará a los pequeños negocios. La industria y el sector creativo también podrían a salir ganando. Un copyright europeo ayudará a combatir la piratería. Y habrá más competencia porque se pretende acabar con los monopolios digitales y una mayor seguridad en la industria digital.

\section{Recomendaciones}

Algunas de las medidas recomendadas para obtener un grado de competitividad en relación con el mercado único digital europeo son:

- Crear normas comunes sobre protección de datos, reformar la normativa sobre telecomunicaciones, modernizar las normas de derechos de autor teniendo en cuenta las nuevas tecnologías, simplificar las normas de protección de los consumidores en compras en línea, facilitar la creación de empresas innovadoras, y mejorar el aprendizaje y las competencias digitales.

- Los países de la región centroamericana deberían estar sujetas a las mismas normas de protección de datos y de los consumidores, independientemente del lugar en que se encuentre su servidor.

- El lanzamiento de un plan de estandarización para identificar y definir las prioridades para sectores como la Salud, el Transporte, el Medioambiente o la Energía y la extensión de servicios de e-Administración para conectar entre sí los servicios públicos a nivel centroamericano.

- La necesidad de una iniciativa público-privada en materia de ciberseguridad. Sobre el tema de la protección de datos en un marco único.

- Eliminar las trabas al acceso a servicios online más baratos de otros países siempre y cuando el bloqueo no esté justificado.

\section{Bibliografía}

Boletín Económico de Información Comercial Española (2010): Transposición de la Directiva de Servicios en España, BICE no 2.990, del 1 al 3 de junio.

Comisión Europea (2011). Digital Agenda Scoreboard. http://ec.europa.eu/information_ society/digitalagenda/scoreboard/index_en.htm 
Comunicación de la Comisión al Parlamento Europeo, al Consejo, al Comité Económico y Social Europeo y al Comité de las Regiones. Acta del Mercado Único - Doce prioridades para estimular el crecimiento y reforzar la confianza: "Juntos por un nuevo crecimiento". COM (2011) 206 final de 13 de abril de 2011.

Comunicación de la Comisión. Estudio Prospectivo Anual sobre el Crecimiento 2012. COM (2011) 815 final, de 23 de noviembre de 2011.

Comunicación de la Comisión: Mejorar la Gobernanza en el Mercado Único. COM (2012) 259 final, de 8 de junio de 2012.

Comunicación dela Comisión.Estudio Prospectivo Anual sobre el Crecimiento 2013. COM (2012) 750 final, de 28 de noviembre de 2012.

Informe al Presidente de la Comisión Europea. "Una Nueva Estrategia para el Mercado Único al Servicio de la Economía y de la Sociedad de Europa”. Monti, Mario. 9 de mayo de 2010.

Informe de la Comisión al Parlamento Europeo, al Consejo, al Banco Central Europeo, al Comité Económico y Social, al Comité de las Regiones y al Banco Europeo de Inversiones. Estado de la integración del Mercado Único 2013. Contribución al Estudio Prospectivo Anual sobre el Crecimiento 2013. COM (2012) 752 final, de 28 de noviembre de 2012.
Monteagudo, J; Rutkows KI, A. y Lorenzani, D. (2012): “El impacto económico de la Directiva de Servicios: un primer análisis tras la implementación”. DG ECFIN de la Comisión Europea. Economic Papers 456/, junio.

Observatorio de Administración Electrónica, (OBSAE). (2012). http://administracionelectronica.gob.es/?_nfpb=true\&_pageLabel=P801924061272299466438\&langPae=es.

ONTSI. (2012). Las TIC en las Empresas y Microempresas Españolas. Edición 2012. http:// www.ontsi.red.es/ontsi/es/estudios-informes/ lastic-en-las-empresas-y-microempresasespa\%C3\%B1olasedici\%C3\%B3n-2012

Ortega, J. (2011): "Programa Europa 2020 y Agenda Digital 2015.eu: hacia un EEES ubicuo, competitivo y excelente”. SCOPEO, El Observatorio de la Formación en Red. Boletín SCOPEO no 55, 15 de diciembre de 2011. En línea: [Consulta: 22/08/2015] - Disponible en: http:// scopeo.usal.es/enfoque-bol-55-programa-europa-2020-y-agenda-digital-2015-eu-hacia-un-eees-ubicuo-competitivo-y-excelente/\#sthash.OrqOyTeA.dpuf. 